\title{
NUEVA REVISTA DE FILOLOGÍA HISPÁNICA
}

AÑO XVI

NÚMS. $1-2$

\section{MUESTRA DEL $A L P I$}

\section{LA $O$ DE $B O C A$}

Entre los 75 mapas que componen el primer volumen del Atlas Lingüistico de la Peninsula Ibérica, el que corresponde a la palabra boca, núm. 26, debe ser uno de los que ofrecen información más pobre y simple. Se emplea tal palabra con la misma forma $y$ sentido tanto en castellano como en catalán, valenciano, gallego y portugués. Se comprende que para el lexicólogo o el fonólogo que sólo atienda a la función de los fonemas el indicado mapa ofrezca escaso interés. Su valor consiste en presentar las modificaciones fonéticas relativas a la pronunciación del vocablo boca en el territorio peninsular.

En las presentes notas el asunto es aún más reducido. Se trata solamente de considerar los datos que el mapa suministra respecto al sonido del fonema $o$ que se destaca en tal palabra con el relieve del acento. La razón de dedicar a vocablo tan común una pregunta en el cuestionario del Atlas fue precisamente la de utilizar su uniformidad gramatical y semántica como marco adecuado para la observación y comparación de los cambios de timbre que pudieran ocurrir en la realización oral de la vocal $o$. En el caso improbable de que en todas partes se registrara una pronunciación idéntica, el hecho no dejaría de ser un testimonio valiosamente informativo.

VARIANTES FONÉTICAS.-Las transcripciones de boca en el mapa. del $A L P I$ no reflejan en modo alguno una pronunciación uniforme. En lo que se refiere al timbre de la $o$, ofrecen las siguientes. variantes:

1) o de timbre medio.

2) o media con tendencia a abierta.

3) o abierta normal.

4) o media con tendencia a cerrada.

5) o cerrada normal.

6) o media con tendencia a mixta.

7) $u$ más o menos abierta.

8) wo con variedad de matices.

9) óu con o semicerrada.

10) úo con o relajada y desvelarizada. 
Se considera como tendencia abierta, cerrada o mixta una inclinación de la vocal que no llega propiamente a la medida semiabierta o semicerrada ni al ordinario tipo mixto. Es comprensible que alguna vez en la impresión de la variante los efectos de la tendencia y de los respectivos tipos intermedios se toquen entre sí. En todo caso no se trata de detalles mínimos fuera del alcance del oído. Tampoco se trata de modificaciones ocasionales ni de la inevitable oscilación de todo sonido articulado. Son rasgos habituales y permanentes que forman parte de la fisonomía o acento particular del habla de cada comarca o región.

Proporción de variantes.-En el conjunto de la Península domina con gran mayoría la pronunciación de boca con o de timbre medio, $41 \%$. A esta modalidad sigue en proporción la $o$ de tendencia abierta, $29 \%$. En tercer lugar figura la variante de tendencia cerrada, $16 \%$; el tipo cerrado normal apenas llega a un $6 \%$, y el propiamente abierto se reduce a $0.5 \%$. Completan la serie ciertos casos en que el fonema etimológico ha mantenido su primitivo sonido de $u$ o ha adquirido forma diptongada.

La $o$ media abarca con mayor o menor grado de densidad todas las zonas de la Península. Por sí sola, uniformemente o alternando con las demás variedades, constituye el fondo más amplio y consistente de la pronunciación de la vocal examinada. Se registra como forma única en los lugares del mapa correspondientes a las provincias de Logroño, Guadalajara, Madrid, Cáceres, Cuenca, Albacete, Murcia, Alicante y Castellón. Con igualdad poco menor aparece en Santander, Álava, Navarra, Soria y Toledo, aparte de aisladas excepciones en favor de la variedad de tendencia abierta. Se extiende asimismo por Zamora, Zaragoza, Teruel y Valencia con escasas discrepancias, las cuales en estas provincias corresponden a la variedad de tendencia cerrada. Ocupa también el primer lugar en Galicia, aunque en proporción menos elevada sobre la variedad de tendencia abierta. Alterna con esta misma variedad por partes iguales en Asturias, Ávila y Ciudad Real. Desciende a papel secundario al lado de las variedades cerradas en Portugal, Cataluña, Alto Aragón y Baleares.

Por su parte, la o de tendencia abierta aparece especialmente establecida en las provincias occidentales de Castilla, en la mitad de Extremadura y en casi toda Andalucía. Ocurre con plena uniformidad en Burgos, Valladolid, Segovia, Badajoz, Huelva, Sevilla, Jaén, Granada, Málaga y Almería. Domina con contadas discrepancias en Córdoba y Cádiz. Comparte con la o media, según se ha indicado, el espacio de Asturias. Figura en lugar inferior en Galicia junto a la misma o media. Se reduce a proporción mínima en Santander y en las demás provincias mencionadas en que el tipo medio desempeña papel casi exclusivo. 
La variedad de tendencia cerrada tiene su más extenso campo de acción en Portugal. A su mismo efecto se suman en esta zona ejemplos relativamente abundantes de plena o cerrada y de los diptongos uó y úo. En el conjunto particular de los lugares portugueses examinados, la proporción de la o plenamente cerrada representa el $21 \%$; la de tendencia cerrada, el $30 \%$, y la de las modificaciones diptongadas y mixtas, el $19 \%$. La $o$ media queda reducida al $25 \%$. Aunque las variedades cerradas y la de timbre medio se reparten por todo el territorio, se puede advertir que los núcleos más densos de las primeras corresponden a los distritos de Viseo y Guarda, en el centro, y a los de Beja y Faro, en el sur, en tanto que las concentraciones más visibles de $o$ media ocurren en los términos de Bragança, Coimbra y Santarem. Las formas diptongadas se dan al norte, en Viana do Castelo, Braga y Oporto.

Al lado opuesto de la Península, las modificaciones más o menos cerradas de la o de boca ocupan lugar preponderante en las provincias catalanas y en el Alto Aragón. Entre los pueblos registrados en esta zona, la variedad de tendencia cerrada representa el $59 \%$ y la plenamente cerrada el $12 \%$. La de la $o$ media se reduce, como en Portugal, al $25 \%$. La pequeña fracción restante corresponde a los ejemplos con $u$, situados exclusivamente en el Rosellón. En los lugares de Gerona y Barcelona, el predominio de la variedad de tendencia cerrada sobre la de timbre medio es mayor que en los de Lérida, Tarragona y Huesca. Los casos de o propiamente cerrada sólo ocurren en el nordeste de Lérida y en el catalán rosellonés.

En Galicia, como queda indicado, alternan la $o$ media y la de tendencia abierta, pero no con el equilibrio que muestran en Asturias ni tampoco con el fuerte predominio que la segunda ejerce en León. En el marco de la región gallega, la modalidad media representa el $60 \%$ y la de tendencia abierta el $36 \%$. Quedan aparte un ejemplo de o cerrada plena en Corcubión, 108, Coruña, y dos de $o$ media con tendencia a mixta en $\mathrm{O}$ Bolo, 146, y A Gradiña, 149, Orense. La ausencia de variedades cerradas, de las que sólo se registra el caso citado, constituye una significativa diferencia entre el gallego y el portugués.

Tipos de Áreas.-De la disposición geográfica de las variantes señaladas resultan áreas uniformes de una sola modalidad de $o$ y áreas mixtas de variedades distintas. Su localización va representada en el grabado adjunto:

1) o media uniforme: línea horizontal.

2) o media predominante sobre tendencia abierta: trazos horizontales.

3) o media predominante sobre tendencia cerrada: trazos horizontales y verticales. 
4) $o$ de tendencia abierta, uniforme: línea punteada.

5) $o$ de tendencia abierta predominante sobre $o$ media: signos circulares.

6) o media y tendencia abierta en igual proporción: línea de trazos y puntos alternos.

7) $o$ de tendencia cerrada predominante sobre o media: línea vertical.

La red de tales áreas ofrece rasgos de consistencia y claridad. Las modalidades de la $o$ media y de la tendencia abierta presentan áreas uniformes. No hay áreas uniformes de o cerrada plena ni de tendencia a cerrada. La tendencia abierta y la tendencia cerrada, cada una de por sí, alternan con la $o$ media, en unos casos como dominantes y en otros como dominadas. Sólo por rara excepción se mezclan una y otra tendencia. De la variedad abierta plena no se hallan más que dos ejemplos en todo el territorio.

Con frecuencia los límites de las áreas coinciden con los de las fronteras políticas y administrativas. Esta coincidencia no es sólo visible entre España y Portugal; son igualmente notorias las divisiones de $o$ media y tendencia abierta entre Murcia y Andalucía, entre Cáceres y Badajoz, entre Zamora y Valladolid y entre Santander y Burgos. Otro ejemplo igualmente definido es el de los límites de $o$ media y de tendencia cerrada entre Valencia y Cataluña. Aparte de la representación de los antiguos reinos, parecen ofrecer estos datos indudable testimonio de la acción lingüística de la unidad provincial.

EnClaves.-Se observan influencias entre áreas inmediatas que revelan contactos de jurisdicción o comunicación. De los cuatro únicos lugares portugueses en que la o de boca fue registrada con tendencia abierta, en contraste con la práctica general del país, el de Salamonde, 205, no lejos de Galicia, el de Rio de Onor, 221, junto a Zamora, y el de Barrancos, 281, frontero a Badajoz, pueden ser atribuidos a influencia de las vecinas áreas españolas. En Zamora, donde rige la $o$ media, el rincón de Sanabria es ejemplo particular de influencias cruzadas entre el leonés, el gallego y el portugués. El caso de San Martín de Castañeda, 338, con o de tendencia abierta, así como el mismo de Rio de Onor, obedecen probablemente a elementos procedentes de Galicia.

En sentido inverso, la $o$ de tendencia cerrada de los lugares zamoranos de Padornelos, 340, y Hermisende, 341, en el mismo rincón sanabrés, debe proceder de Portugal. Este mismo origen cabe suponer en los casos de tendencia cerrada y de $o$ cerrada plena que aparecen en línea directa desde Sanabria a Asturias junto a la raya de Galicia: Ponte de Rey, 324, en León, y Salgueiras, 30o, As Campas, 301, Freal, 302, y Cuantas, 323, en Asturias.

Contactos semejantes se pueden ver en Santander, donde la 


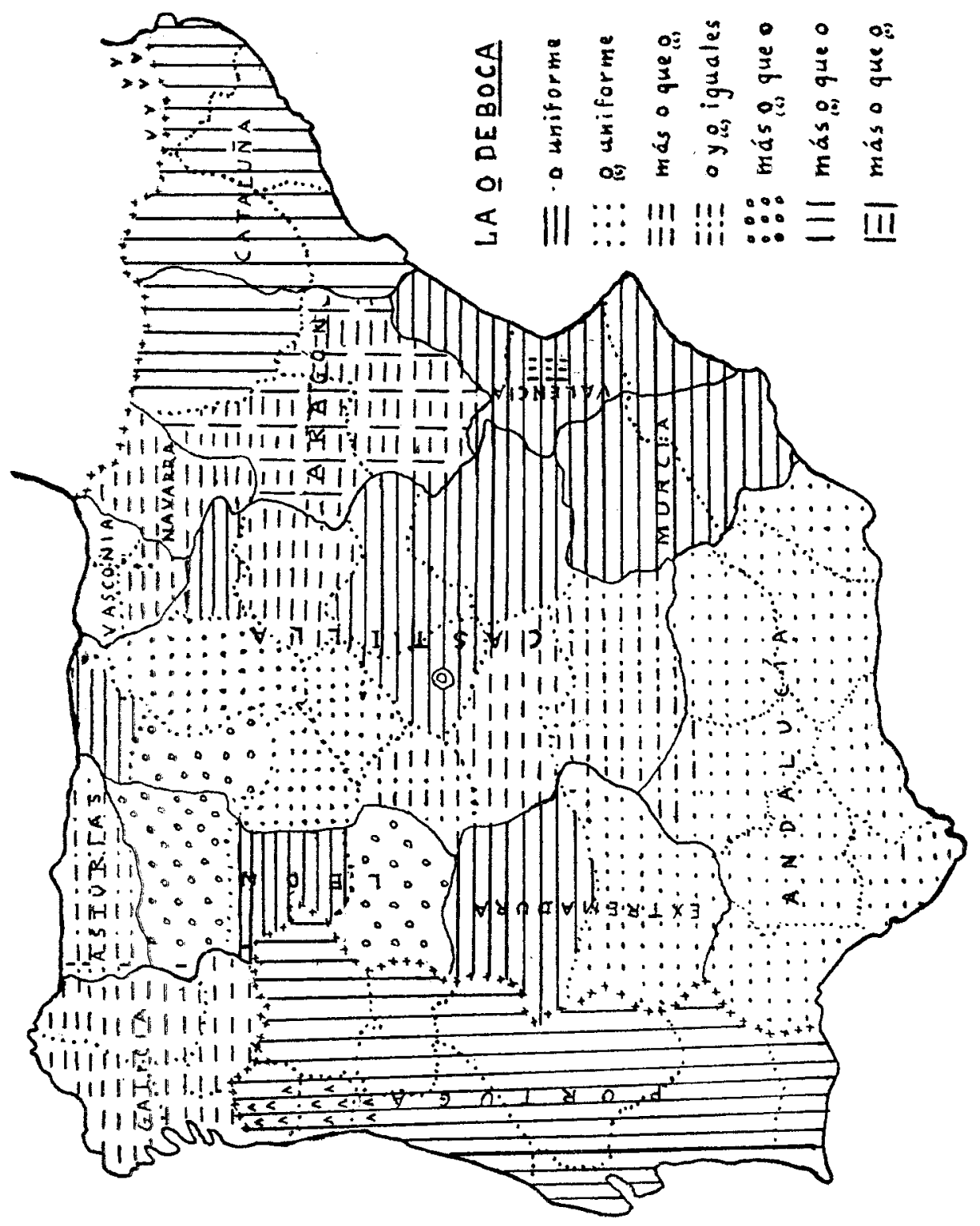


uniformidad de la $o$ media sólo ofrece las excepciones con tendencia abierta de Balbacienta, 403, y Veguilla, 4og, en los límites con Vizcaya, así como en Álava, en la que sólo el condado de Treviño, 424, y el lugar de Ozaeta, 431, vecino a Guipuzcoa, ambos de tendencia abierta, se apartan de la variedad media del resto de la provincia. Tanto en el caso de Santander como en el de Álava es posible imaginar alguna relación con el vasco, cuya o ordinaria, por lo menos en guipuzcoano, tiende generalmente al timbre abierto.

En el lugar de Galbe de Sorbes, 459, Guadalajara, la $o$ de tendencia abierta, única discrepancia en la uniformidad provincial de la $o$ media, indica probable parentesco con la modalidad de tendencia abierta general en la vecina Segovia. Los dos puntos de $o$ media en la parte de Córdoba sobre el Guadalquivir, Villaviciosa, 5o3, y Adamuz, 504, siguen el ejemplo del inmediato Ciudad Real, a diferencia de la general tendencia abierta andaluza. Los lugares valencianos que forman un islote de tendencia cerrada y de cerrada plena en el centro de la uniforme o media de esta región -Turis, 772, Polinyá de la Ribera, 774, Massalavés, 775, Anna, 777, Moixent, 780 - dan base al supuesto de un posible fondo fonético de influencia aragonesa.

No es de extrañar que la tendencia abierta alcance alguna vez en Andalucía el grado de plena abertura, registrado en Alcalá de los Gazules, 534, Gádiz. Lo extraño es que no se repita tal ejemplo en Andalucía ni en otras áreas de la misma tendencia y que, por el contrario, la única vez que vuelve a encontrarse otro caso de plena $o$ abierta ocurra en medio tan distinto como el de Cornellá de Llobregat, 740, bajo la jurisdicción de la tendencia cerrada de Cataluña. Otros casos aislados y enigmáticos son el de Pardilho, 242, en las costas de Aveiro, Portugal, con tendencia abierta; el ya citado de Corcubión, 108, en las costas de La Coruña, con plena $o$ cerrada; los de O Bolo, 146̂, y A Gudĩáa, 149, en Orense, cerca de Sanabria, con $o$ de tendencia mixta, y el de Ferreira de Alcántara, 365, Cáceres, en la frontera portuguesa, con diptongo ou de o semicerrada y breve $u$ semivocal.

La mayor alteración de la o es la diptongación que se manifiesta principalmente en los distritos antes mencionados del norte de Portugal bajo las formas básicas de uó y úo con diversos matices que afectan especialmente al timbre de la $o$. No es una diptongación de rasgos regularmente definidos. Aparece como un fenómeno vacilante que cambia de uno a otro lugar. En los detalles de la minuciosa y elaborada transcripción fonética se advierte el esfuerzo del investigador para representar la impresión de tan movibles efectos. Las diversas modificaciones recogidas en los 16 puntos en que aparece tal diptongación, constituyen tres grupos distintos: uno a base de uo en que el primer elemento, semivocal, muestra mayor o menor 
desarrollo y en que la o varía entre media y cerrada; otro sobre la misma base en que la $o$ adopta la calidad mixta de vocal central que a veces se oye como $a$ o $e$ neutras e incoloras, y otro, de base $u$, en que la $o$ secundaria y con frecuencia la misma $u$ apoyada por el acento presentan también por su parte el timbre impreciso de la vocal mixta. Aparte de la especial concentración de estos diptongos en los términos de Viana do Castelo, Braga y Oporto, otro ejemplo figura en Vilarinho de Samardã, 219 , Vila Real, y dos más se extienden hasta Sobrado de Paiva, 241, Aveiro, y Sobreiro, 272, Lisboa. Por su carácter y localización representan tales diptongos un arduo interrogante en la dialectología portuguesa.

Evolución vertical.-La o media y la de tendencia cerrada ocupan casi sin excepción la mitad oriental de la Península. Castilla se reparte entre $o$ media a saliente y tendencia abierta a poniente. La $o$ cerrada plena y la tendencia cerrada del norte de Cataluña, $u$ en el Rosellón, se atenúa en el sur del condado y se desvanece al extenderse el idioma hasta tierras de Alicante. La tendencia cerrada del Alto Aragón se reduce en Zaragoza y Teruel y se pierde en el reino de Murcia. Análogo proceso se observa de norte a sur en las provincias orientales de Castilla, donde la o media, prácticamente uniforme desde la Rioja a la Mancha, alterna con tendencia abierta en Toledo y Ciudad Real y se borra en Andalucía. Salta a la vista la semejanza entre tal desarrollo y los avances de la lengua en la recuperación y repoblación del territorio.

El orden de las modalidades de la $o$ en las provincias castellanas de occidente y en las leonesas no responde a la misma evolución indicada. Tanto la variedad media como la de tendencia abierta parecen arrancar de fuentes cantábricas. La primera sobresale en Santander, y una y otra comparten el principado de Asturias. En su expansión hacia el sur, se mezclan y entrecruzan a lo largo de su curso. Por alguna oculta razón, la o media penetró hasta la frontera de Portugal por los términos de Zamora y Cáceres, rompiendo la unidad que de otro modo hubiera podido mostrar, desde Burgos a Badajoz y Andalucía, la variedad de tendencia abierta.

Alguna relación cabe suponer, sin embargo, entre las secciones del norte, centro y sur de esta amplia zona de uniforme o predominante tendencia abierta, no obstante los núcleos interpuestos de Zamora y Cáceres. Es de pensar que, aparte de la probable transición de $o$ media a tendencia abierta entre Ciudad Real y Córdoba, la gran extensión alcanzada por esta tendencia en Badajoz y Andalucía acaso obedezca de manera principal a la presencia en gradó predominante de gentes procedentes de León y del oeste de Castilla en la repoblación de estas tierras meridionales.

La uniforme presencia de la tendencia abierta en Burgos, Valladolid y Segovia y su supremacía en León, Palencia y Salamanca, 
rechazan el supuesto de que la vocal evolucionara hacia timbre más abierto al pasar desde las regiones relativamente frías del norte a las más templadas del sur. El testimonio del portugués, con no menos densidad de variedades cerradas en el sur que en el norte, se opone a esa misma conjetura.

Las zonas del catalán, del aragonés y del castellano oriental excluyen visiblemente la variedad de tendencia abierta. Las del castellano occidental y del leonés rechazan la tendencia cerrada. En lo que se refiere al presente caso, por lo menos, los resultados no coinciden con la idea que suele atribuir cierta condición de mayor claridad al vocalismo levantino. Resulta asimismo inesperado el hecho de que el tiembre de la vocal sea más abierto en Burgos que en Madrid. En el punto examinado, Galicia se asocia al leonés y al castellano occidental, no al portugués. El único centro en que las tendencias abierta y cerrada conviven en proporciones semejantes, alternando además con la o media, son las Baleares. Con clara diferenciación, en Mallorca, los lugares del norte y oeste de la isla muestran modalidades cerradas, en contraste con la tendencia abierta de los del sur y este. Finalmente, de acuerdo con la dialectología general de la $\mathrm{Pe}$ nínsula, la configuración de las áreas en el norte es más estrecha y variada que en el sur.

\section{LA $E$ DE $C E P A$}

No podrá menos de ofrecer interés la comparación de los resultados obtenidos del examen de la o de boca con los de la $e$ de cepa que figura en el $A L P I$ con el núm. 42. La confrontación de los mapas de ambas palabras dará a conocer hasta qué punto se corresponden entre sí sus respectivas circunstancias geográficas.

Desde luego, el vocablo cepa, como instrumento comparativo, no constituye para la vocal $e$ un marco tan regular e invariable como el que boca proporciona a la $o$. Se puede decir que el concepto de cepa sólo es familiar y frecuente en las regiones en que se cultiva la vid. Coincide morfológicamente en español y portugués; en catalán y valenciano carece de la vocal final, cep. Además, en varios lugares aparece bajo forma masculina, cepo, aunque con la misma significación.

Por otra parte, la red geográfica de cepa es quebrantada de vez en cuando por la interferencia de algunos sinónimos: videira, cepeira y podón, en puntos de Galicia; tronco, bara, padrão, tronço, toro y pé de vide, en Portugal; cabeza de parra, en un lugar de Badajoz; caña, en otro de Córdoba, y troncón, en otro de Sevilla. Las zonas de denominación uniforme, sin sinónimos, son las de Castilla, Navarra, Aragón y Murcia (cepa), y las de Cataluña, Valencia, Baleares y Rosellón (cep).

Otras palabras del $A L P I$ con $e$ acentuada, como abeja, cabeza, 
cereza, eje, etc., responden a otros propósitos y experimentan también diversos cambios.

VARIANTES Fonéticas.-La $e$ de cepa figura en las transcripciones del $A L P I$ con las siguientes variantes:

1) $e$ de timbre medio.

2) $e$ de tendencia abierta.

3) e abierta normal.

4) $e$ ultraabierta.

5) $e$ de tendencia cerrada.

6) e cerrada normal.

7) $e$ mixta de timbre variable.

8) $i \dot{e}$ con diversos matices.

9) ie con $e$ cerrada.

Proporaión de variantes.-Aparece a la cabeza con destacada superioridad la $e$ de timbre medio, 66\%. La sigue la variedad de tendencia abierta, $20 \%$. Ocupa el tercer lugar la $e$ propiamente abierta, $8 \%$. La $e$ cerrada y la de tendencia cerrada apenas suman un $2 \%$. Las demás variedades representan fracciones aún menores.

La $e$ media se caracteriza por su extensión peninsular. Se ve firmemente establecida en Castilla, Asturias, León, Navarra, Aragón y Murcia. Sólo dos lugares con tendencia abierta forman excepción entre los 75 examinados en las provincias castellanas; tres de esa misma tendencia se cuentan entre los 29 correspondientes a León, Zamora y Salamanca; ningún punto se aparta del ordinario tipo medio entre los 55 registrados en territorio navarro, aragonés y murciano. La proporción de la $e$ media en Portugal representa el $70 \%$ del conjunto de los pueblos estudiados en este país. En la región valenciana esta proporción sube al $77 \%$. De Extremadura, cae bajo el dominio de la $e$ media la provincia de Cáceres, de Cataluña la de Lérida, y de Andalucía la de Almería y parte de la de Córdoba.

La $e$ de tendencia abierta aparece establecida de manera general en Badajoz, Huelva, Sevilla, Cádiz y Málaga; ejerce superioridad sobre la $e$ media en Galicia, en las provincias andaluzas de Granada y Jaén y en el catalán del Rosellón; reduce su representación en Valencia; se limita a casos excepcionales, como se ha indicado, en Castilla, León y Cataluña, y no muestra ejemplo alguno en Asturias, Navarra, Aragón ni Baleares.

La $e$ plenamente abierta domina en Cataluña con gran superioridad sobre el tipo de timbre medio, y es en Baleares la forma ordinaria y corriente, con la excepción de Mahón, 803, en Menorca, en que fue registrada con sonido ultraabierto.

De la modalidad de tendencia cerrada sólo se cuentan cuatro casos aislados, de los cuales se hallan dos en Portugal, uno en Asturias y otro en Extremadura. Los tres únicos ejemplos de $e$ plena- 
mente cerrada se dan en Portugal en los distritos de Coimbra y Leiria. Las modalidades diptongadas y las de timbre mixto ocurren asimismo exclusivamente en territorio portugués.

Tipos de Áreas.-Como en el caso de la o de boca, se distinguen áreas uniformes en que actúa una sola variedad de $e$ y áreas mixtas en que intervienen diferentes variedades. Se representa su disposición en el adjunto grabado:

1) e media uniforme: línea horizontal.

2) $e$ media predominante sobre tendencia media: trazos horizontales.

3) $e$ de tendencia abierta, uniforme: línea punteada.

4) $e$ de tendencia abierta predominante sobre $e$ media: signos circulares.

5) $e$ abierta uniforme: línea diagonal.

6) $e$ abierta y $e$ media en igual proporción: trazos diagonales.

Al lado de la extensa área de la $e$ media, presentan también áreas uniformes la $e$ abierta plena y la de tendencia abierta. Las áreas mixtas combinan estas dos variedades con la $e$ media. El conjunto peninsular aparece menos subdividido que en el mapa de boca. Las áreas mixtas se reducen en el caso de cepa a las provincias gallegas y a partes de Cataluña, Valencia y Andalucía. No ocurren áreas mixtas de $e$ media y tendencia cerrada semejantes a las que ocupan considerable parte del cuadro de la $o$.

La extensión de la e media en la pronunciación de cepa no sólo incluye el área correspondiente a la o de este mismo tipo, sino también las provincias de Castilla y León ocupadas por la o de tendencia abierta, además de las partes ya indicadas de Andalucía, y asimismo el Alto Aragón y la provincia de Lérida, que en el mapa de boca muestran $o$ de tendencia cerrada. El portugués, que en la pronunciación de boca da preferencia a esta misma tendencia, se suma al castellano en el predominio de la $e$ media de cepa.

Otras varias observaciones se deducen de la comparación entre ambas palabras. En una y otra coincide la clara línea divisoria entre la variedad media de Cáceres y la tendencia abierta de Badajoz. El área mixta de Galicia mantiene consistentemente su delimitación regional. La tendencia abierta andaluza reduce en la $e$ de cepa su extensión y su uniformidad. El contraste más visible lo constituye la $e$ abierta de Cataluña, debida probablemente a la sílaba trabada de $c e p$, frente a la tendencia cerrada de la o de boca en esta misma región.

EnClaves.-Los puntos desplazados, en desacuerdo con su propio medio, son en cepa relativamente escasos. El lugar de Campanario, 370 , único con $o$ media en la uniforme tendencia abierta de Badajoz, sugiere por su proximidad a Cáceres el posible origen de su discre- 
pancia. Freal, 302, en el noroeste de Asturias, confirma con su $e$ de tendencia cerrada la singularidad ya advertida con motivo de su $o$ semicerrada en la pronunciación de boca. Los demás lugares leoneses y zamoranos con $o$ cerrada en los límites con Galicia desde Asturias a Sanabria, aparecen convertidos en cepa a la uniformidad de la $e$ media.

Los ejemplos portugueses de diptongación de la $e$, con variedad de efectos en la pronunciación del diptongo, corresponden a los mismos distritos en que se registró la diptongación de la $o$ de boca, pero afectan a menor número de lugares. Tales modificaciones alrededor de ciepa se encuentran en Marinhas, 207, Braga; en Paço de Rei, 2 16, Oporto, y en Vilarinho de Samardã, 219, Vila Real. Una variedad de ceipa, con cierto timbre de redondeamiento labial del diptongo, se hace notar en Oleiros, 253, Castelo Branco.

La forma $c e p$ con $e$ media de la provincia de Lérida se extiende a puntos vecinos de Huesca: La Pobla de Roda, 612, Benabarre, 613, y Sant Esteva de Llitera, 614. Una línea común cabe suponer entre los ejemplos del masculino cepo que, con la misma significación del femenino, descienden desde Aragón a Andalucía: en Huesca, Fonz, 615; en Valencia, Pedralba, 768; Godalleta, 771, Dos Aguas, 773, y Anna, 777; en Murcia, la Pinilla de Caravaca, 562, Abanilla, 566, Tiñosa, 567, Purias, 568, y Cabo de Palos, 570; en Almería, Taberno, 555, y Mojácar, 560; en Granada, Zujar, 547. Los indicados pueblos valencianos en que se dice cepo o sepo, en lugar del común valenciano sep, forman parte del mismo centro en que la o de boca constituye un islote de timbre cerrado dentro del área de o media general en la región. El timbre cerrado, que rechaza la idea de procedencia castellana, sugiere, como se ha indicado con respecto a la o de boca, la procedencia aragonesa del habla de estos lugares, a la que sus naturales consideran como "castellano chapurreado".

\section{RESUMEN}

El mero examen de la vocal o en el mapa de boca y de la $e$ en el de cepa descubre íntimos aspectos, en su mayor parte insospechados, de la geografía lingüística peninsular. Otros mapas proporcionarán información de más relieve y sustancia, sobre todo los referentes a puntos principales de fonética histórica, de sinonimia geográfica y de léxico etnográfico.

El estrecho tejido de las áreas señaladas en ambas palabras refleja un ámbito peninsular densamente vivido y trabajado. Las líneas de tales áreas responden sin duda a circunstancias y episodios cuya historia invita a minuciosa investigación. No deben ser casuales ni siquiera los ejemplos solitarios en discrepancia con el me- 
dio en que se hallan. Hechos que aquí acaso parezcan minúsculos podrán adquirir mayor volumen con el testimonio de los mapas de otras palabras.

La $o$ y la $e$ se corresponden fonológicamente. Su posición en boca y cepa las rodea de circunstancias semejantes. Sus cuadros geográfico-fonéticos coinciden básicamente en el predominio de sus respectivos tipos de timbre medio. Apoyados sobre este eje, uno y otro fonema se han movido entre los extremos abierto y cerrado, si bien la inclinación de cada uno de ellos difiere considerablemente respecto a tales extremos.

Otros puntos de significativa coincidencia son, como ha podido verse, la persistente diferenciación entre Cáceres y Badajoz, la definida individualidad de Galicia, el predominio de las modalidades de tendencia abierta en las provincias del sur, la diptongación de $e$ y $o$ en la misma comarca del norte de Portugal y la concurrencia de ciertos rasgos peculiares en el mismo grupo de pueblos valencianos.

En la compleja geografía de boca se observa que la o de timbre medio y la variedad de tendencia abierta son las dos modalidades que compiten más extensamente en el campo del español peninsular. La $o$ de tendencia abierta, con base en Burgos y con apoyo de gran parte de Castilla la Vieja y de León, cuenta además en su favor con la amplia adhesión de Andalucía. La o media, por su parte, con base en Madrid y con apoyo de Castilla la Nueva y Levante, está asistida por la ventaja de su propio timbre medio, acorde con el equilibrio del sistema vocálico del español común o normal.

El mapa de cepa presenta una situación más simple y despejada, aunque hubiera podido parecer natural que una palabra tan corriente y fanniliar como boca favoreciera la uniformidaci de su vocal acentuada en mayor grado que la menos frecuente cepa. Es de pensar que la $e$, por virtud de su propia articulación palatal, de mecanismo más preciso que el de la o, y por la misma claridad de su timbre, se preste más que esta última a la corriente igualadora que a base de los tipos medios parece actuar en el fondo como ideal fonético del idioma. Favorece además a la $e$ en este sentido el figurar junto a la $a$ al frente del repertorio fonológico de las lenguas peninsulares, con gran ventaja sobre la proporción de la $o$.

Evolución gradual de norte a sur se advierte en la $e$ de cepa a lo largo de las provincias mediterráneas. Gerona y Barcelona muestran uniforme variante abierta; en Tarragona disminuye esta variedad ante la intervención de la $e$ media; en Castellón y Valencia se refuerza la $e$ media mientras que la modalidad abierta se reduce a mera tendencia, y en Alicante esta tendencia se borra dejando el campo a la $e$ media que se extiende uniformemente a través de Murcia y Almería. 
El proceso indicado, de $e$ abierta a media, contrasta con el movimiento inverso de la $o$ de boca en esas mismas zonas, pero ambos casos vienen a confirmar, como se ve, su común convergencia hacia sus respectivos tipos medios. El progreso de la $e$ media se acentúa con el testimonio de parte de Andalucía, donde, según se ha notado, la modalidad de tendencia abierta, con menos resistencia que la correspondiente variedad de $o$, se ha retirado de este a oeste y de norte a sur en beneficio del predominante tipo indicado.

La $o$ media, aunque extensamente establecida en el país, se ve aún enfrentada y contenida por amplias zonas de sólida inclinación cerrada o abierta. Con desarrollo más amplio y uniforme, la $e$ media ha igualado la mayor parte del territorio, exceptuando a Galicia, Andalucía occidental, Rosellón y Baleares, donde la tendencia abierta persiste con relativa firmeza.

Los mapas de boca y cepa, como todos los del $A L P I$, representan el habla de las personas iletradas en los pueblos y aldeas rurales. Es de suponer que los cuadros que podrían resultar de la pronunciación de esas mismas palabras entre las personas instruidas ensancharían el dominio de los tipos medios de $e$ y $o$, aunque realmente las variedades y tendencias señaladas no muestran visible connotación de orden cultural.

La ausencia del habla urbana no es la única limitación del ALPI. Adolece de las mismas restricciones que afectan a los atlas lingüísticos generales de otros países. Para abarcar la Península en una exploración realizable en un plazo no desmesurado, que por graves acontecimientos se prolongó durante largo tiempo, la empresa tuvo que reducir el número de lugares estudiados, de personas examinadas en cada lugar y de materias incluidas en el cuestionario. Corresponde a los atlas particulares de regiones o comarcas aplicar a la encuesta una red de distancias más cortas, comprender en la investigación personas de distintos niveles de cultura y edad, y extender el cuadro del léxico etnográfico a toda clase de sectores y actividades tradicionales.

En lo que el $A L P I$ no se impuso limitación alguna fue en el empeño de representar la imagen fonética de las palabras con la mayor precisión posible, de sugerir con los recursos de una refinada transcripción todo matiz perceptible en el timbre y articulación de los sonidos, de ofrecer en sus mapas para futuros estudios los múltiples reflejos del habla actual en los lugares campesinos, como querríamos que hubiera sido posible recoger y representar la pronunciación de cualquier época pasada.

Transcripciones someras son retratos deficientes e incompletos. En este sentido la minuciosidad del $A L P I$ no parecerá excesiva a los lingüistas interesados en descifrar las modificaciones y cambios de los sonidos en relación con las peripecias históricas de las gentes 
que los hayan manejado. Los que sólo busquen los rasgos generales siempre podrán prescindir de lo que les sobre.

En todo caso, en su propósito de exactitud, el $A L P I$ no se guió por principios de escuela que hayan sufrido alteración o hayan perdido actualidad, sino por puras y permanentes exigencias de su misión informadora. Con método menos riguroso, el secreto fonético, recogido en cada mapa, de variedades, tendencias y hábitos, como los presentados en esta muestra, no todos apreciables a simple vista para personas inexpertas, pero fáciles de reconocer para cualquier dialectólogo debidamente preparado, sería probable que como tal secreto hubiera quedado sin advertir ni registrar.

Tomás Navarro

Northampton, Mass. 